\title{
Caracterización psicométrica, motora y funcional en niños con parálisis cerebral
}

\author{
RAÚL ESCOBAR H. ${ }^{1}$, ALICIA NÚÑEZ F. ${ }^{1}$, ÁNGELA HENAO V. ${ }^{2}$, \\ JAIME CERDA L. ${ }^{3}$, ALFONSO COX M. ${ }^{4}$, MARCELA MIRANDA M. ${ }^{5}$ \\ 1. Neurólogo Pediatra. División de Pediatría, Unidad de Neurología, Laboratorio de Neurorehabilitación y Enfermedades \\ Neuromusculares, Facultad de Medicina, Pontificia Universidad Católica de Chile. \\ 2. Residente de Pediatría, Facultad de Medicina, Pontificia Universidad Católica de Chile. \\ 3. Pediatra, Especialista en Salud Pública, Departamento de Salud Pública Facultad de Medicina, Pontificia Universidad \\ Católica de Chile. \\ 4. Psicólogo, Laboratorio de Neurorehabilitación y Enfermedades Neuromusculares, Facultad de Medicina, \\ Pontificia Universidad Católica de Chile. \\ 5. Kinesióloga, Laboratorio de Neurorehabilitación y Enfermedades Neuromusculares, Facultad de Medicina, \\ Pontificia Universidad Católica de Chile.
}

\begin{abstract}
Psychometric, motor and functional description in children with cerebral palsy

Introduction: Cerebral Palsy (CP), the most frequent cause of chronic motor disability among children, is often associated to other alterations of cerebral function, among them cognitive function. International publications show up to $70 \%$ of children with CP have normal intelligence (IQ). Objective: Determine IQ and motor function in a group of children with CP. Methodology: Prospective descriptive study of 34 children with CP, classified according to type. Psychometric and motor tests were applied. Results: Of these children, 44\% presented tetraparetic CP, 21\% diparetic, 35\% hemiparetic. Among tetraparetic children, 33\% showed normal IQ, 14\% mild Mental Retardation (MR), 53\% severe MR. Among diparetic children, normal IQ, mild MR, severe MR showed in a frequency of $57 \%, 14 \%$ y $29 \%$ respectively. Children with hemiparesis showed normal IQ in 92\% and mild MR in 8\%. Motor function was usually proportional to IQ. Conclusion: In the full population studied, 59\% of children showed normal IQ. Children with hemiparetic CP present better IQ, those with tetraparetic CP showed worst cognitive levels. Children with better IQ presented better motor function.

(Key words: IQ, Disability, Motor Function, Cerebral Palsy, Neurorehabilitation, Psychometric Tests).

Rev Chil Pediatr 2011; 82 (5): 388-394

\section{RESUMEN}

Introducción: La Parálisis Cerebral (PC), causa más frecuente de discapacidad motora crónica en la infancia, se asocia frecuentemente a otras alteraciones de la función cerebral, entre ellas alteraciones cognoscitivas. Publicaciones extranjeras describen que hasta un $70 \%$ de los niños con PC tienen coeficiente intelectual (CI) normal. Objetivo: Determinar CI y Función Motora en un grupo de niños con PC. Metodología: Estudio
\end{abstract}

Trabajo recibido el 21 de marzo de 2011, devuelto para corregir el 02 de mayo de 2011, segunda versión el 28 de junio de 2011, aceptado para publicación el 20 de septiembre de 2011.

Correspondencia a:

Raúl Escobar H.

E-mail: rescobar@med.puc.cl 
prospectivo descriptivo de 34 niños con PC. Se les clasificó según tipo de PC, se aplicó test psicométrico y se realizó medición del grado de función motora. Resultados: 44\% tenían PC tetraparética, 21\% diparética y 35\% hemiparética. Los niños con tetraparesia tenían en un 33\% CI normal, 14\% RM leve y 53\% RM severo; los niños con diparesia: CI normal, RM leve y RM severo en 57\%, 14\% y 29\% respectivamente; los niños con hemiparesia tenían CI normal en el 92\% y RM leve en 8\%. En cuanto a función motora, los niños con mejor CI tenían mejor grado de función motora. Conclusión: El 59\% del grupo estudiado presentó CI normal. Los niños con PC hemiparética tienen mejor CI y los niños con PC tetraparética tienen peor CI. Los niños con mejor CI tienen mejor grado de función motora.

(Palabras clave: Coeficiente intelectual, discapacidad, función motora, parálisis cerebral, neurorehabilitación, test psicométricos).

Rev Chil Pediatr 2011; 82 (5): 388-394

\section{Introducción}

La Parálisis Cerebral (PC) es la causa más frecuente de discapacidad motora en la infancia. Bajo este término se incluye un amplio grupo de síndromes no progresivos que tienen en común alteración del tono, postura y movimiento, secundarios a lesiones o malformaciones producidas durante el período de desarrollo temprano del cerebro ${ }^{1,2}$. A pesar de los avances en el cuidado perinatal y neonatal, la incidencia de PC no ha variado con los años, estimándose una cifra de 2 a 3 por 1000 recién nacidos vivos ${ }^{3-6}$. Sin embargo, se ha demostrado que el tratamiento multidisciplinario con base en neurorehabilitación temprana y el abordaje precoz con detección y tratamiento oportuno de las comorbilidades, ha permitido mejorar y aumentar la sobrevida en este grupo de niños ${ }^{7,8}$.

Si bien, como se mencionó anteriormente, el principal síntoma de la PC es el trastorno del movimiento y la postura, otras manifestaciones del compromiso del Sistema Nervioso Central (SNC) pueden estar presentes en este grupo de pacientes, tales como: alteración de la sensación y percepción, trastornos de la comunicación, alteración del comportamiento, convulsiones y dificultades cognoscitivas y neuropsicológicas globales o específicas ${ }^{9-11}$.

Se estima que hasta un $70 \%$ de los niños con PC tienen un Coeficiente Intelectual (CI) normal o cercano a la normalidad ${ }^{12-14}$, existiendo una relación entre la severidad del compromiso cognoscitivo y el tipo de PC, y entre la intensidad del trastorno motor y el nivel de funcionalidad ${ }^{13,15}$. Sin embargo, son pocos los estudios que evalúan el CI en niños con $\mathrm{PC}^{13,16}$, esto es debido a la complejidad que existe al aplicar los test psicométricos en este grupo de niños, dado por las limitaciones físicas, neurosensoriales y del lenguaje que éstos presentan $^{17-19}$. Estudios previos han reportado que un 15 a $20 \%$ de los niños con PC tienen gran dificultad en responder los test de inteligencia habituales, por lo que, frecuentemente la evaluación cognoscitiva de éste grupo de niños es parcial o basada en la evaluación clínica $^{20}$. En la Unidad de Neurorehabilitación y Enfermedades Neuromusculares Pediátricas de la Pontifica Universidad Católica de Chile, se atienden rutinariamente niños portadores de PC, siendo la determinación de su CI un elemento trascendente en la planificación de su neurorehabilitación.

El objetivo principal del presente trabajo es caracterizar el Coeficiente Intelectual de un grupo de niños con Parálisis Cerebral, que asisten a la Unidad de Neurorehabilitación y Enfermedades Neuromusculares Pediátricas de la Pontificia Universidad Católica de Chile. Como objetivo secundario está el relacionar el tipo de Parálisis Cerebral con Coeficiente Intelectual y el grado de Función Motora con Coeficiente Intelectual.

\section{Pacientes y Método}

Estudio prospectivo con reclutamiento de pacientes desde agosto de 2007 a julio de 2010. Se incluyeron pacientes con diagnóstico 
de PC, con edades entre los 4 y 16 años, que tuviesen consentimiento informado y firmado por sus padres y/o cuidadores y acudiesen en forma regular a la Unidad. Se excluyeron niños con discapacidad visual y/o auditiva severa, que impidiera realizar los test psicométricos disponibles; aquellos que recibían depresores del SNC, y los que no cooperaron para la realización del test. A cada uno de los niños ingresados se le hizo un registro de los datos demográficos. A todos se les realizó examen clínico por neurólogo. Se clasificó el tipo de PC utilizando la clasificación neuroanatómica o topográfica, que las diferencia de acuerdo a la extensión del compromiso, no se consideró en la clasificación el tipo de vía comprometido. Se les clasificó en PC Tetraparética, PC Diparética y PC Hemiparética.

A todos los pacientes se les realizó test psicométrico por psicólogo entrenado en evaluación de niños con PC. Los test psicométricos empleados fueron los test de medición de inteligencia de Wechsler, validados en Chile: WIPPSI (para niños entre edades de 4 a 6 años) y WISC-R (entre 6 años 6 meses y 16 años 11 meses). Cada test incluye evaluación de escala verbal y escala manual. De acuerdo al puntaje obtenido se agrupó a los pacientes en diferentes rangos de CI (21): normal (70-109), retardo metal (RM) leve (60-69), RM moderado (5059) y RM profundo (<49).

Se determinó el grado de función motora mediante las escalas de función motora gruesa, GMFCS ("Gross Motor Function Classification System”) y GMFM ("Gross Motor Function Meassure”) ${ }^{22-23}$. La escala GMFCS clasifica en 5 niveles, de I a V, de menor a mayor grado de limitación. Evalúa el desempeño funcional durante la sedestación y la marcha, evaluando el déficit funcional, necesidad de elementos de ayuda para movilizarse y la calidad del movimiento. Para el presente estudio, reagrupamos los diferentes niveles en: nivel I como nivel I, nivel II y nivel III como nivel IIIII, y nivel IV y nivel V como nivel IV-V. La otra escala utilizada fue la GMFM, instrumento diseñado para medir cambios en las habilidades motrices gruesas en niños con PC. Este instrumento evalúa al niño en cinco dimensiones: 1) en decúbito y rodando; 2) en sedente;
3) en gateo y sobre rodillas; 4) de pie; 5) caminando, corriendo y saltando. Da un puntaje de 0 a 100 para cada posición, entre más alto mejor función de cada unos de los movimientos ${ }^{24}$. La evaluación fue realizada por kinesióloga entrenada. El puntaje final se expresó como el promedio total de cada uno de los puntajes alcanzados en cada posición.

Para el análisis de los datos se utilizó el programa estadístico SPSS calculando la media porcentaje del total de cada variable analizada. En el caso de comparación de dos variables categóricas se utilizó el test de $\chi^{2}$ considerando como diferencia estadística significativa una $\mathrm{p}<0,05$.

El estudio fue aprobado por el Comité de Ética de la Pontificia Universidad Católica de Chile.

\section{Resultados}

Un total de 38 niños fue reclutado para este estudio, siendo incluidos finalmente 34, 50\% de sexo femenino y $50 \%$ sexo masculino. El rango de edad fue entre los 4 años y 16 años 2 meses, promedio de 8 años y 7 meses. De los 4 niños excluidos del estudio, uno no completó el total de los test, uno por no interés posterior de los padres en continuar y dos por ser portadores de una PC teatraparética espástica grave, secundaria a encefalopatía hipóxica isquémica post PCR, con nivel de conciencia mínima.

En cuanto al CI, el 59\% tuvo un CI en rango normal, $12 \% \mathrm{CI}$ en rango $\mathrm{RM}$ leve y $29 \%$ CI en rango RM profundo (tabla 1).

De los 34 niños, el 44\% tenía una tetraparesia, el 21\% diparesia y el 35\% hemiparesia (Tabla 1). En cuanto a la distribución de los diferentes grados de CI según el tipo de PC: en el grupo de niños con tetraparesia, 33\% tenía un CI normal, 14\% RM leve y 53\% RM severo. El grupo de niños con diparesia, 57\% tenían CI normal, 14\% RM leve y 29\% RM severo. En el grupo de niños con hemiparesia, 92\% tenían CI normal, y 8\% RM leve (figura 1).

El promedio de la escala de CI verbal (CIV) y de la escala de CI manual (CIM) según el tipo de PC, fue de 56 en CIV y 49 en CIM para el grupo de niños con tetraparesia. Para el gru- 
po de niños con diparesia, el promedio de CIV fue 76 de CIM de 64. Mientras que el grupo de niños con hemiparesia tuvo un promedio de CIV de 103 y de CIM 88 (figura 2).

Tabla 1. Distribución de los 34 niños por tipo de PC, $\mathrm{Cl}$ y nivel de GMFCS

\begin{tabular}{|llll|}
\hline $\begin{array}{l}\text { Tipo } \\
\text { de PC }\end{array}$ & Tetraparética & Diparética & Hemiparética \\
\hline & $44 \%$ & $21 \%$ & $35 \%$ \\
\hline Cl & $n=15$ & $n=7$ & $n=12$ \\
\hline & Normal & RM leve & RM profundo \\
& $59 \%$ & $12 \%$ & $29 \%$ \\
\hline GMFCS & $\mathrm{n}=20$ & $\mathrm{n}=4$ & $\mathrm{n}=10$ \\
\hline & Nivel I & Nivel II-III & Nivel IV-V \\
& $41 \%$ & $24 \%$ & $35 \%$ \\
\hline & $\mathrm{n}=14$ & $\mathrm{n}=8$ & $\mathrm{n}=12$ \\
\hline
\end{tabular}

PC: Parálisis Cerebral; Cl: Coeficiente intelectual; GMFCS: Sistema de Clasificación de Función Motora Gruesa; RM: Retardo mental.
Según el sistema de clasificación GMFCS, $41 \%$ se ubicaba en el Nivel I, $24 \%$ en el nivel II-III y $35 \%$ en el nivel IV-V (tabla 1).

En cuanto a la relación entre CI y el grado clínico de función motora (GMFCS): los niños con un nivel I de función motora tuvieron CI normal en el $86 \%$ de los casos y RM leve en un 14\%. Los niños con nivel II-III de función motora tuvieron CI normal en el 50\% de los casos, RM leve en el 13\% y RM profundo en el 37\%. Los niños con nivel IV-V de función motora tuvieron CI normal en el 33\% de los casos, RM leve en el 9\% y RM severo en el $58 \%$ de los casos (figura 3 ).

En cuanto a la relación del promedio de la medición de la función motora gruesa (GMFM) con CI, los niños con un CI normal tuvieron un puntaje promedio de función motora gruesa de 79 puntos, los niños con RM leve 68 puntos y los niños con RM severo tuvieron un promedio
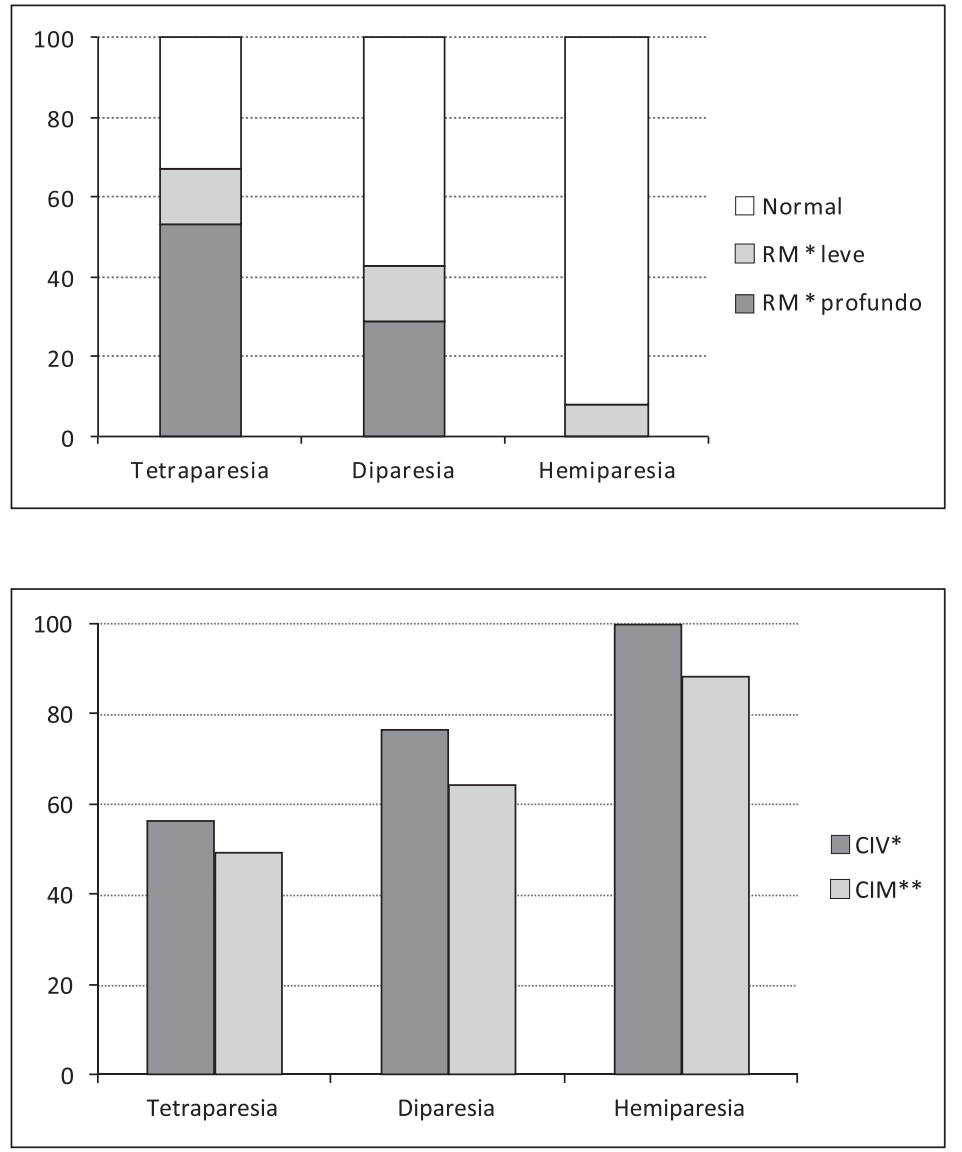

Figura 1. Relación entre tipo de parálisis cerebral y coeficiente intelectual. *RM: retardo mental.
Figura 2. Promedio de capacidad intelectual verbal y manual, según tipo de parálisis cerebral. *Capacidad intelectual verbal. **Capacidad intelectual manual. 

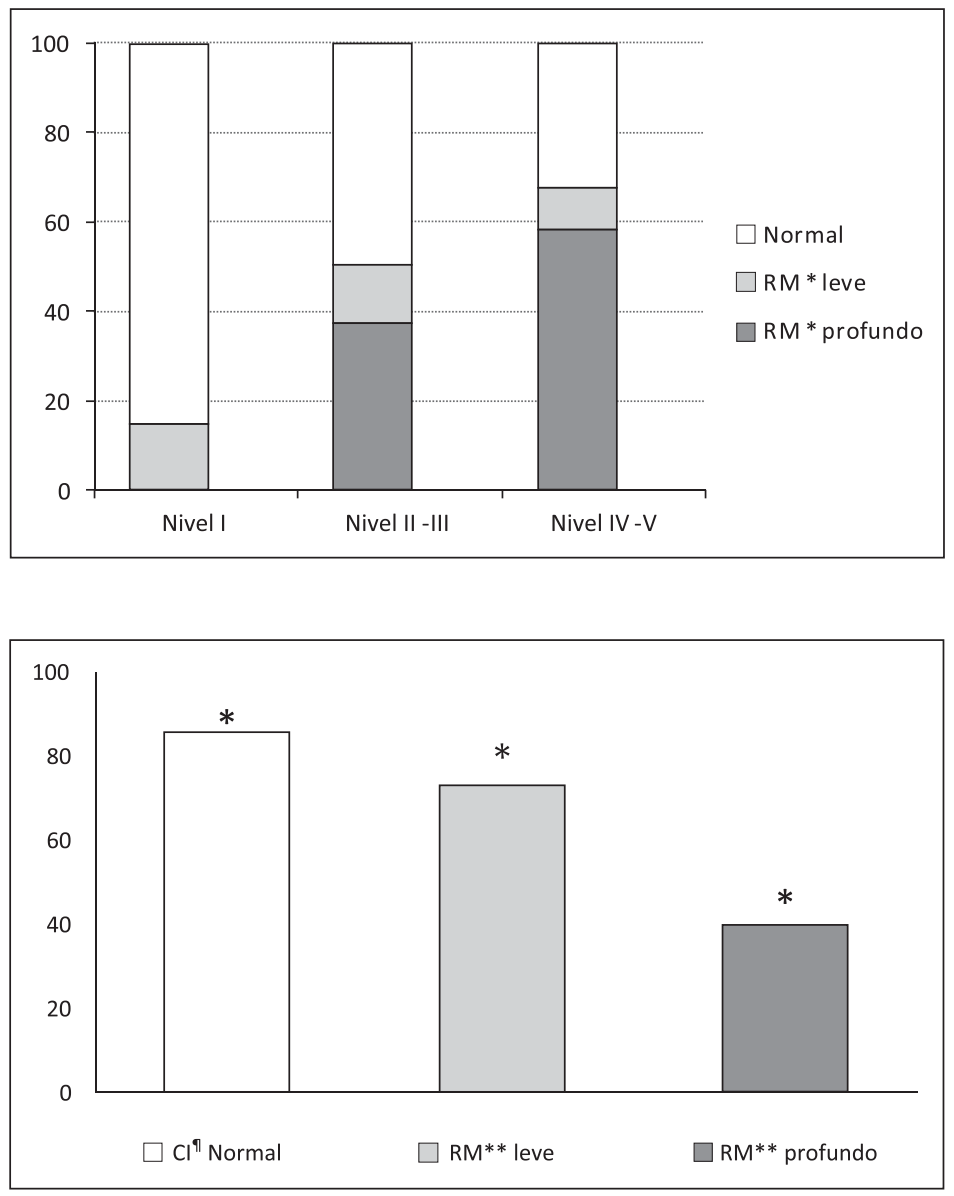

Figura 3. Relación entre capacidad intelectual y "GMFCS". *RM: Retardo mental. "GMFCS": Sistema de clasificación de la función motora gruesa.

Figura 4. Promedio GMFM según coeficiente intelectual. ${ }^{*} p<0,005$; ${ }^{9} \mathrm{CI}$ : Coeficiente intelectual; **RM: Retardo metal. GMFM: Sistema de clasificación de función motora gruesa. de 37 puntos, siendo estas diferencias estadísticamente significativas (figura 4).

\section{Discusión}

En el grupo de niños con PC ingresados en nuestro estudio, hubo predominio de niños con PC tetraparética, presumiblemente debido a la mayor proporción de pacientes graves que atiende nuestra Unidad. A cada uno de ellos se les logró caracterizar el coeficiente intelectual, se relacionó el tipo de PC con el CI y se relacionó el grado de función motora con CI. Nuestros resultados son cercanos a lo descrito en la literatura, donde más del 50\% de los niños con PC tiene un CI normal, siendo más afectados aquellos niños con tetraparesia y menos afectados el grupo de niños con hemiparesia $^{12,13,15}$. Encontramos en este estudio que los niños con mejor CI se agrupan en aquellos con mejor funcionalidad motora o más bien aquellos niños con mejor funcionalidad motora tienen mejor $\mathrm{CI}^{16,19}$. Una de las dificultades más importantes a la hora de realizar la cuantificación del coeficiente intelectual en niños con Parálisis Cerebral, es la aplicación de los test existentes, pues ellos están validados para niños sin déficit motor. Los test psicométricos actuales, validados en nuestro país, evalúan dos áreas fundamentales: verbal y manual. En este grupo de niños, al ser el compromiso motor el pilar fundamental en el diagnóstico de PC, los tiempos y las técnicas a implementar deben ser adaptadas considerando este factor. Algo parecido ocurriría en la evaluación verbal, pues muchos de estos niños presentan alteraciones del lenguaje en distinto grado lo que en teoría dificultaría o limitaría los resultados. 
Lo anterior, explica la relevancia de que el evaluador tenga el entrenamiento y la experiencia adecuada para aplicar estos test, condición que se cumplió en la evaluación de los pacientes aquí reportados.

$\mathrm{Al}$ analizar los puntajes de la escala manual y escala verbal, el promedio de los puntajes en todos los grupos fue mayor en la escala verbal que en la manual, lo que confirma entonces el mayor compromiso motor. Relacionado a lo anterior, es el resultado que obtuvimos del CI en el grupo de niños con tetraparesia, pues aunque el 53\% de ellos tienen un RM severo, el 33\% tiene un CI normal, indicando que aunque estén muy limitados desde el punto de vista motor, su función cognoscitiva se encuentra indemne, por lo que, en este grupo de niños la neurorehabilitación motora tendría aún una mayor relevancia, puesto que una mejoría en ella mejoraría su independencia. Al igual que lo encontrado en nuestro trabajo, otros estudios realizados hasta ahora concuerdan en que los niños con PC tetraparética tienen un grado de compromiso intelectual mayor que los niños con hemiplejia ${ }^{17}$.

Si bien es cierto, evaluaciones neurológicas de laboratorio usuales, como estudios electroencefalográficos $\mathrm{o}$ de neuroimágenes ${ }^{18}$, y funcionales como las aquí mostradas pueden orientar respecto al grado de compromiso cognoscitivo en niños con PC, lo certero es efectuar evaluaciones psicométricas formales, para establecer claramente el nivel cognoscitivo de este tipo de pacientes. Especialmente por el hecho que se ha demostrado que cuanto más precoz se hace la intervención en estos niños, mejor es el resultado que se alcanza ${ }^{17,25}$. Los objetivos en el manejo de estos niños debe ser optimizar al máximo sus capacidades tanto en el aspecto motriz, interacción social, desarrollo cognoscitivo e independencia. El tratamiento óptimo debe ser multidisciplinario, de tal manera de detectar y manejar a tiempo las co-morbilidades que se puedan presentar. Como parte de las recomendaciones de evaluación de los niños con PC se debe incluir la evaluación de su desempeño cognoscitivo, puesto que esto permite planificar de manera más adecuada el apoyo que deberá recibir el niño y la forma de comunicarse con él' ${ }^{26,27}$.
Finalmente, podemos resumir nuestros resultados en que el 59\% del grupo estudiado presentó CI normal. Los niños con PC hemiparética tienen mejor $\mathrm{CI}$, mientras que los niños con PC tetraparética tienen peor CI. Los niños con mejor CI tienen mejor grado de función motora.

Si bien el número de pacientes incluido en este estudio es pequeño, podemos plantear por los resultados, que la caracterización cognoscitiva de este grupo de niños es factible de realizar, aunque se requiere de herramientas adecuadas para lograr este objetivo, pero especialmente experiencia en la aplicación de éstas.

La evaluación psicométrica debe ser parte de la evaluación general de todo niño con PC, para poder así implementar y adecuar el plan de neurorehabilitación de forma integral.

\section{Referencias}

1.- Koman A, Paterson B, Shilt J: Cerebral Palsy. Lancet 2004; 363: 1619-31.

2.- Yelin B: Diagnóstico temprano de la parálisis cerebral. Rev Neurol 1997; 25: 725-8.

3.- Krigger K: Cerebral Palsy: An Overview. Am Fam Physician 2006; 73: 91-100.

4.- Nelson K, Grether J: Cerebral Palsy in low birthweight infants: etiology and strategies for Prevention. MRDD Research Reviews 1997; 3: 112-7.

5.- Stanley F: Survival and Cerebral Palsy in low birthweight Infants: implications for perinatal care. Paediatr Perinat Epidemiol 1992; 2: 298-310.

6.- Hagberg B, Hagberg G, Beckung E: Changing panorama of Cerebral Palsy in Sweden. Prevalence and origin in the birth year period 1991-94. Acta Paediatr 2001; 90: 271-7.

7.- Strauss $D$, Shavelle $R$ : Survival in cerebral palsy in the last 20 years: signs of improvement?. Dev Med Child Neurol 2007; 49: 86-92.

8.- Legido A, Katsetos C: Parálisis cerebral: nuevos conceptos etiopatogénicos. Rev Neurol 2003; 36: 157-65.

9.- Shapiro B: Cerebral Palsy: A Reconceptualization of the Spectrum. J Pediatr 2004; 145: S3-S7.

10.- Bax M, Goldstein M, Rosenbaum P, Leviton A, Paneth $N$ : Proposed definition and classification of cerebral palsy, April 2005: Introduction. Dev Med Child Neurol 2005; 47: 571-6.

11.- Bottcher L: Children with Spastic Cerebral Palsy, their cognitive functioning, and social participation: a review. 
Child Neuropsychol 2010; 16: 209-28.

12.- Murphy C, Boyle C, Schendel D, Decoflé P, YearginAllsopp M: Epidemiology of Mental retardation in Children. MRDD Research Reviews 1998; 4: 6-13.

13.- Paneth N, Stark R: Cerebral Palsy and Mental Retardation in relation to indicators of perinatal asphyxia. Am J Obstet Gynecol 1983; 147: 960-6.

14.- Robertson C, Finer N: Term Infants with hipoxicischemic encephalopathy: outcome at 35 years. Dev Med Child Neurol 1985; 27: 473-84.

15.- Beckung E, Hagberg G: Neuroimpairments, activity limitations, and participation restrictions in children with cerebral palsy. Dev Med Child Neurol 2002, 44; 309-16.

16.- Ashwal S, Russman B, Blasco P, et al: Practice Parameter: Diagnostic assessment of the child with cerebral palsy. Report of the Quality Standards Subcommittee of the American Academy of Neurology and the Practice Committee of the Child Neurology Society. Neurology 2004; 62: 851-63.

17.- Trauner D, Ballantyne A, Friedland S, Chase C: Disorders of affective and linguistic prosody in children after early unilateral brain damage. Ann Neurol 1996; 39: 361-7.

18.- Dammann O, Kuban K, Leviton A: Perinatal Infection, Fetal Inflammatory Response, White Matter Damage and Cognitive Limitations in Children Born Preterm. MRDD Research Reviews 2002; 8: 46-50.

19.- Hutton J, Pharoah P: Effects of cognitive, motor, and sensory disabilities on survival in cerebral palsy. Arch
Dis Child 2002; 86: 84-9.

20.- Sigurdardottir S, Eiriksdottir A, Gunnarsdottir E, Meintema $M$, Arnadottir $U$, Vik T: Cognitive profile in young Icelandic children with cerebral palsy. Dev Med Child Neurol 2008; 50: 357-62.

21.- Ramírez V, Rosas $R$ : Test de Inteligencia para niños de Wechler. Manual de administración y puntuación. Normas de administración chilena. $4^{\text {a }}$ ed. Santiago: Ediciones Universidad Católica de Chile; 2010.

22.- Palisno R, Rosenbaum P, Walter S, Rusell D, Gallupi $B$ : Development and reliability of a system to classify gross motor function in children with CP. Dev Med Child Neurol 1997; 39: 214-23.

23.- Palisno R, Rosenbaum P, Bartlett D, Livingston M: Content validity of the expanded and revised Gross Motor Function classification System. Dev Med Child Neurol 2008; 50: 744-50.

24.- Rosenbaum P, Walter S, Hanna S: Prognosis for gross motor function in cerebral palsy. JAMA 2002; 288: 1357-63.

25.- White-Koning M, Grandjean H, Colver A, Arnaud C: Parent and professional reports of the quality of life of children with cerebral palsy and associated intelellectual impairment. Dev Med Child Neurol 2008; 50: 618-24.

26.- Waters E, Davis A, Mackinnon A, et al: Psychometric properties of the quality of life questionnare for children with CP. Dev Med Child Neurol 2007; 49: 49-55.

27.- Stiers $P$, Vandenbussche $E$ : The dissociation of perception and cognition in children with early brain damage. Brain and Dev 2004; 26: 81-92. 